\title{
Abstracts from the 2nd International Norges Teknisk-Naturvitenskapelige Universitet (NTNU) Symposium: Day 2-biomarkers in precision diagnosis and treatment
}

Published online: 21 October 2019

C) Springer Nature Limited 2019

\author{
Current and Future Clinical Biomarkers of Cancer: \\ From diagnosis to immunotherapy—why is precision \\ medicine so difficult?
}

14 and 15 June 2018, Trondheim, Norway

All of the presentations from the meeting both days can be seen and listened to here (https://vimeo.com/showcase/ 5456812).

Editors $^{1}$ and Organizers ${ }^{2}$ : Ana I. Robles ${ }^{1}$, Magne Børset $^{2}$, Anders Sundan ${ }^{2}$, Anne-Marit Sponaas ${ }^{1,2}$, Oluf Dimitri Røe $\mathrm{e}^{1,2}$

Conflict of interest: ODR has received honorary from NOVARTIS for a scientific lecture. AR, MB, AS and AMS declare that they have no conflict of interest.

This Symposium aimed at presenting research on clinically useful biomarkers and emerging concepts of diagnosis, prognosis, prediction and treatment of cancer. These abstracts from day 2 mainly focused on solid tumors and biomarkers of immunotherapy, chemotherapy and early diagnosis, in which experts from Europe, USA, and Australia reviewed the field and shared their most recent advances. This meeting will quickly become a welcoming forum for an interdisciplinary dialog on translational studies aimed at improving cancer diagnosis and treatments.

Sponsorship: The symposium was sponsored by the Norwegian University of Science and Technology (Norges Teknisk-Naturvitenskapelige Universitet, NTNU), HUNT (Helse-unders $ø$ kelsen i Nord-Trøndelag) research center, the Norwegian Research Council and Immunological Society of Norway, Norwegian Society of Biochemistry, Norwegian Cancer Society and AstraZeneca, BMS, MSD, Amgen, Celgene, Pfizer, Roche and EISAI. All content was reviewed and approved by the authors and organizers, which held full responsibility for the abstract selections. Publication of the supplement was sponsored by Norwegian University of Science and Technology (NTNU).
Immunotherapy, practice and biomarkers

D2-01

Lung cancer-from best supportive care to immunotherapy. The biomarkers tale

Fred R. Hirsch ${ }^{1,2}$

${ }^{1}$ University of Colorado Anschutz Medical Campus, Aurora, CO, USA; ${ }^{2}$ Mount Sinai Health System, NY, USA

In less than two decades, lung cancer has emerged from a "non-treatable" disease with dismal prognosis (1) to a role model for the application of precision medicine and immunotherapy to solid tumors. Reduced smoking and advances in lung cancer screening and treatment have all contributed to a steady decline in lung cancer associated mortality during this time period (2). However, key factors behind the significant progress in therapy and prognosis have also been the identification of molecular subtypes of this cancer and the development of targeted therapies and immunotherapies (3). As the understanding of lung cancer has moved from histological-based to molecular-based classifications, the identification of new targets, and particularly biomarkers to select patients who may benefit from targeted therapies, has been crucial (4). In his keynote presentation, Fred Hirsch, former CEO of the International Association for the Study of Lung Cancer (IASLC), reviewed landmark studies that led to advances in lung cancer therapy, from chemotherapy to precision medicine, and, most recently, immunotherapy. Subgroups of patients with lung cancer can today be treated with several 
generations of targeted therapies, e.g. patients with tumors harboring $E G F R$ mutations and $A L K$ - gene rearrangements as well as other genomic abnormalities, and for many patients we are rapidly moving towards a "chronic treatment paradigm". For some subgroups of non-small cell lung cancer (NSCLC) e.g. ALK-rearrangement positive patients with advanced disease, the 5-year survival has been reported to be about $40 \%$. Furthermore, most recent advances in immunotherapy have been demonstrated in NSCLC patients presenting with advanced disease. Despite this progress, obstacles still exist in many countries regarding implementation of molecular testing and access to new targeted therapies. Using lung cancer as a case example, Hirsch dvelved into the challenges that hinder the implementation of precision medicine including, inter- and intra-tumor heterogeneity, the need for liquid biopsies (plasma/urine-based), monitoring treatment effect, acquired resistance, and predictive assays (for immunotherapy).

Conflict of interest: FH has served at the advisory board or as consultant for BMS, AstraZeneca, Merck, Roche/ Genentech, Loxo/Bayer, Abbvie, Novartis, Helsinn, HTG Molecular, Biocept, and received lecture fees from Roche and AstraZeneca. He has received grants by Amgen, Biodesix, Mensana, Rain Therapeutics and Merck. He has one patent without royalties through the University of Colorado: EGFR copy number and Protein as Predictive Biomarkers in Lung Cancer.

\section{References}

1. Schiller JH, Harrington $\mathrm{D}$, Belani $\mathrm{CP}$, Langer $\mathrm{C}$, Sandler A, Krook J, et al. Comparison of four chemotherapy regimens for advanced non-small-cell lung cancer. N Engl J Med. 2002;346:92-8.

2. Siegel RL, Miller KD, Jemal A. Cancer statistics, 2019. CA Cancer J Clin. 2019;69:7-34.

3. Hirsch FR, Scagliotti GV, Mulshine JL, Kwon R, Curran WJ, Jr, Wu YL, et al. Lung cancer: current therapies and new targeted treatments. Lancet. 2017;389:299-311.

4. Vargas AJ, Harris CC. Biomarker development in the precision medicine era: lung cancer as a case study. Nat Rev Cancer. 2016;16:525-37.

\section{D2-02.}

\section{The role of pathology in the development of immunotherapy clinical trials}

\section{Robert Anders ${ }^{1}$}

${ }^{1}$ Johns Hopkins University, Baltimore, MD, USA
The level of programmed death-ligand 1 (PD-L1) in tumor cells is regarded as a putative biomarker for response to antibodies that target its interaction with the programmed death 1 (PD-1) receptor on T cells (1). However, clinical implementation of PD-L1 as a biomarker has been compromised by lack of assay standardization and poor performance, and there is an urgent need for better biomarkers to predict response to immune-based therapy (2). Robert Anders gave an overview of the current knowledge regarding predictive biomarkers of response to anti-PD-1 treatment and the work in his lab to develop better markers for this purpose. In a landmark proof-ofconcept clinical study of colorectal patients receiving antiPD-1 treatment, Anders and colleagues showed that patients with mismatch repair-deficient tumors (detected as microsatellite instability) exhibited significantly better response to treatment than patients with mismatch repair-proficient tumors (3). This result has now been expanded to show the efficacy of PD-1 blockade in patients with mismatch repair-deficient tumors regardless of the cancers' tissue of origin, across 12 different tumor types. In all, microsatellite instability and expression of PD-L1 in the tumor tissue had a positive predictive value of around 50\% and a negative predictive value of about $15 \%$ for response to treatment (4). Beyond the tumor cells themselves, other features of the tumor immune microenvironment, including immune infiltration, were found to be important predictors of response to anti-PD-1 blockade. When staining tumor tissue from patients with microsatellite instability for PD-L1, they found that PD-L1 was expressed, but not uniformly across the tumor and not by the tumor cells themselves. PD-L1 was expressed by tumor-associated macrophages at the tumor margin, at the invasive front of the tumor $(5,6)$. While PD-L1 alone was insufficient for predicting response of patients with merkel cell carcinoma (MCC), a rare aggressive skin cancer, to anti PD-1 therapy, they found that close proximity of PD-1-positive cells and PD-L1positive cells (within $20 \mu \mathrm{m}$ ) was associated with clinical response (7). In other tumors, Anders' group is developing lymphocyte mapping strategies, which allow them to analyze the localization of different $\mathrm{T}$ cell populations relative to tumor tissue. Early results indicate that some tumors exclude $\mathrm{T}$ cells whereas others are infiltrated by $\mathrm{T}$ cells. Work is in progress to see how this feature may correlate to patient outcome. Much work is still needed to develop new and better markers, and existing markers must be integrated and analyzed in combination.

Conflict of interest: RA has served at the advisory board or as consultant for BMS, AstraZeneca and Merck.

\section{References}

1. Topalian SL, Hodi FS, Brahmer JR, Gettinger SN, Smith DC, McDermott DF, et al. Safety, activity, and immune correlates of anti-PD-1 antibody in cancer. $\mathrm{N}$ 
Engl J Med. 2012;366:2443-54.

2. Nishino M, Ramaiya NH, Hatabu H, Hodi FS. Monitoring immune-checkpoint blockade: response evaluation and biomarker development. Nat Rev Clin Oncol. 2017;14:655-68.

3. Le DT, Uram JN, Wang H, Bartlett BR, Kemberling $\mathrm{H}$, Eyring $\mathrm{AD}$, et al. PD-1 blockade in tumors with mismatch-repair deficiency. $\mathrm{N}$ Engl J Med. 2015:372:2509-20.

4. Le DT, Durham JN, Smith KN, Wang H, Bartlett BR, Aulakh LK, et al. Mismatch repair deficiency predicts response of solid tumors to PD-1 blockade. Science. 2017;357:409-13.

5. Taube JM, Klein A, Brahmer JR, Xu H, Pan X, Kim $\mathrm{JH}$, et al. Association of PD-1, PD-1 ligands, and other features of the tumor immune microenvironment with response to anti-PD-1 therapy. Clin Cancer Res. 2014; 20:5064-74.

6. Taube JM, Galon J, Sholl LM, Rodig SJ, Cottrell TR, Giraldo NA, et al. Implications of the tumor immune microenvironment for staging and therapeutics. Mod Pathol. 2018;31:214-34.

7. Giraldo NA, Nguyen P, Engle EL, Kaunitz GJ, Cottrell TR, Berry $\mathrm{S}$, et al. Multidimensional, quantitative assessment of PD-1/PD-L1 expression in patients with Merkel cell carcinoma and association with response to pembrolizumab. J Immunother Cancer. 2018;6:99.

\section{D2-03.}

\section{Expression and co-expression of checkpoint signaling receptors cluster of differentiation 39 (CD39) and programmed death 1 (PD-1) are highly elevated in intra- tumoral immune cells in non-small cell lung cancer}

\author{
Anders Tondell, ${ }^{1,2}$ Markus Haug ${ }^{1,2}$ \\ ${ }^{1}$ Norwegian University of Science and Technology, Trond- \\ heim, Norway; ${ }^{2}$ St. Olavs University Hospital, Trondheim, \\ Norway
}

In advanced non-small cell lung cancer (NSCLC), tumor immune escape mechanisms may collaborate in a nonoverlapping fashion, so that targeting one immune checkpoint may not be sufficient to relieve tumor-induced immunosuppression. Checkpoint inhibition of the programmed death 1 (PD-1) signaling pathway remains without effect in $\sim 80 \%$ of the patients. Recently, extracellular adenosine, generated by the ectonucleotidases cluster of differentiation 39 and 73 (CD39, CD73) on immune cells, was recognized as an immunosuppressive checkpoint mediator (1). CD39 degrades extracellular adenosine tri-phosphate (ATP) released from dead or decaying cells to adenosine mono-phosphate (AMP), which is then converted to adenosine by CD73. The contribution and cooperation between different tumor immune escape mechanisms in NSCLC remains unknown. Anders Tøndell described a study aimed at investigating the phenotype and expression of markers of immunosuppression on key immune cell subtypes in NSCLC, which used tumor and adjacent healthy lung tissue samples from surgicallyresected NSCLC patients. Immune cells were extracted by combining enzymatic and mechanical disaggregation followed by density gradient centrifugation. Expression of markers of immunosuppression was investigated by flowcytometry. The fractions of $\mathrm{CD}^{+}$and $\mathrm{CD} 4^{+} \mathrm{T}$ cells coexpressing CD39 and PD-1 cells were highly increased in tumor compared to healthy lung tissue (median: $34.6 \%$ versus $4.3 \%, p=0.0005$ on $\mathrm{CD} 8^{+} \mathrm{T}$ cells and $34.9 \%$ versus $10.7 \%, p=0.0010$ on $\mathrm{CD}^{+}{ }^{+} \mathrm{T}$ cells). Tumor tissue also showed increased frequencies of $\mathrm{CD} 39^{+} \mathrm{PD}-1$-negative $\mathrm{CD}^{+}$and $\mathrm{CD}^{+} \mathrm{T}$ cells compared to healthy lung tissue. PD- $1^{+} \mathrm{CD} 39$-negative $\mathrm{CD}^{+}$and $\mathrm{CD}^{+} \mathrm{T}$ cells were significantly increased in tumor, although present in both tumor and healthy lung tissue. Regarding $\mathrm{T}$ cells with a regulatory phenotype, the total frequency of $\mathrm{FoxP}^{+} \mathrm{CD}^{+}$ regulatory $\mathrm{T}$ cells as well as co-expression of $\mathrm{CD} 39$ and PD-1 on these cells were significantly elevated in tumor compared to adjacent healthy tissue (Figure 1). Similar to tumor-infiltrating $\mathrm{T}$ cell subsets, frequencies of CD39expressing tumor-associated macrophages were more than three-fold increased in tumor compared to lung tissue. This data demonstrated that NSCLC tumor-infiltrating immune cells consistently show elevated expression and coexpression of markers of immune suppression on $\mathrm{CD} 4^{+}$, $\mathrm{CD}^{+}$, forkhead box P3 $\left(\mathrm{FoxP}^{+}\right.$) regulatory $\mathrm{T}$ cells and tumor-associated macrophages, indicating signaling pathways for immunosuppression and tumor immune-escape acting simultaneously.

Conflict of interest: The authors declare that they have no conflict of interest.

\section{References}

1. Allard B, Longhi MS, Robson SC, Stagg J. The ectonucleotidases CD39 and CD73: Novel checkpoint inhibitor targets. Immunol Rev. 2017;276:121-44.

Figure 1. Immune cell phenotype fractions in NSCLC and adjacent healthy lung tissues. Co-expression of CD39 and PD- 1 on $\mathrm{CD}^{+}{ }^{+}$and $\mathrm{CD} 8^{+} \mathrm{T}$ cells and FoxP3 on $\mathrm{CD} 4^{+}$ $\mathrm{T}$ cells extracted from tumor or adjacent lung tissue. AC, Adenocarcinoma; SCC, Squamous Cell Carcinoma 


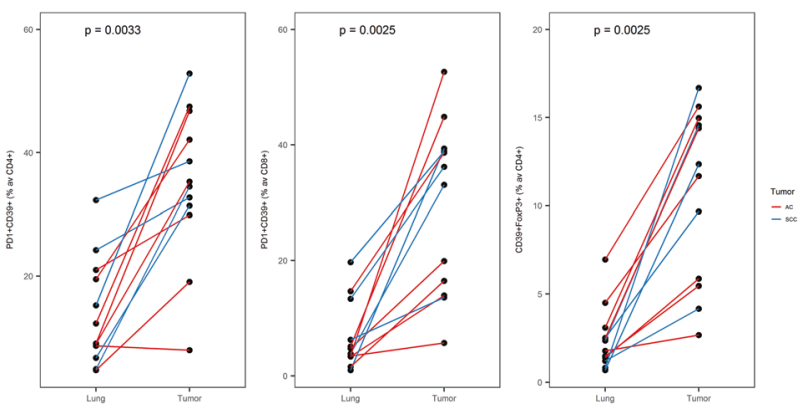

Early diagnosis

D2-04.

DNA methylation and associated gene expression prior to lung cancer diagnosis: A study of pre-diagnostic blood samples from the Norwegian Women and Cancer Cohort

\section{Therese Nøst ${ }^{l}$}

${ }^{1}$ The Arctic University of Norway, Troms $\phi$, Norway

Tobacco smoking is the number one cause of lung cancer, however up to $15 \%$ of lung cancer cases occur in never smokers and the incidence of lung cancer in never smokers appears to be increasing (1). Although the epigenetic effects of smoking exposure that may represent predisposition to lung cancer and other diseases have been characterized (2) smoking-independent biomarkers of lung cancer risk remain relatively unexplored. Therese Nøst described an epigenome-wide search within a nested case-control study (131 cases, 129 controls) in a Norwegian prospective cohort of women designed to investigate the role of smoking exposure in disease-related associations (3). They found 25 $\mathrm{CpG}$ sites associated with lung cancer risk. Stringent adjustment for smoking exposure using a comprehensive smoking index (CSI) revealed two associated $\mathrm{CpG}$ sites, the cg10151248 (PC, CSI-adjusted OR $=0.36$ [0.25-0.52] per standard deviation change in methylation) and the cg13482620 (B3GNTL1, CSI-adjusted $\quad \mathrm{OR}=0.33$ [0.22-0.050]). Importantly, analysis of never smokers only and of an independent cohort of smoking-discordant twins confirmed the independence of these markers from smoking exposure. Gene expression profiles related to the smokingindependent $\mathrm{CpG}$ sites showed enriched pathways different from those related to smoking-dependent $\mathrm{CpG}$ sites and could possibly reflect immunological changes in blood prior to cancer diagnosis. This study illustrates the potential of blood-derived DNA methylation as a biomarker of lung cancer risk, independent of tobacco exposure and related to distinct biological pathways (the work in this abstract was published subsequently as a full paper in Scientific Reports) (3).

Conflict of interest: TN has received grant support from The University of Troms $\varnothing$ and the Norwegian Research Council.

\section{References}

1. Pelosof L, Ahn C, Gao A, Horn L, Madrigales A, Cox J, et al. Proportion of Never-Smoker Non-Small Cell Lung Cancer Patients at Three Diverse Institutions. J Natl Cancer Inst. 2017;109.

2. Joehanes R, Just AC, Marioni RE, Pilling LC, Reynolds LM, Mandaviya PR, et al. Epigenetic signatures of cigarette smoking. Circ Cardiovasc Genet. 2016;9:436-47.

3. Sandanger TM, Nost TH, Guida F, Rylander C, Campanella G, Muller DC, et al. DNA methylation and associated gene expression in blood prior to lung cancer diagnosis in the Norwegian Women and Cancer cohort. Sci Rep. 2018;8:16714.

\section{D2-05.}

Metabolomics signatures in serum years before thoracic cancer: the Nord-Trøndelag Health Study/ Helseundersøkelsen i Nord-Trøndelag (HUNT)

Christina Chatzipantsiou ${ }^{1}$, Oluf Dimitri Røe $e^{2,3,4}$

${ }^{1}$ University of Crete, Heraklion, Greece; ${ }^{2}$ Levanger Hospital, Levanger, Norway; ${ }^{3}$ Norwegian University of Science and Technology, Trondheim, Norway; ${ }^{4}$ Aalborg University Hospital, Aalborg, Denmark

The propensity for lung cancer biomarkers to be detected in serum of high-risk groups, such as smokers at an early disease stage, is an area of high interest for clinicians, since early diagnosis is paramount to effective treatment. Metabolic profiling can help in the development of noninvasive diagnostic tests while also providing insights for the underlying disease pathology. Christina Chatzipantsiou, of Oluf Dimitri Røe's research group, discussed metabolomic profiling conducted within the Nord-Trøndelag Health (HUNT) Study, a longitudinal population health study in Norway (1). Pre-diagnostic albumin-depleted serum samples from 48 future lung cancer and mesothelioma patients and 48 matched controls, obtained from the HUNT Research Centre's Biobank were screened for metabolites by an liquid chromatography-mass spectrometry (LC-MS) untargeted metabolomics approach. The cohort consisted of 12 of each of adenocarcinoma, squamous cell carcinoma, small-cell lung cancer and 
mesothelioma along with their respective matched controls. All subjects included in the study were cancer-free 5 years after blood sampling; lung cancer subjects (adenocarcinoma, squamous cell carcinoma, small-cell lung cancer) were smokers. Instrument raw data were exported and processed in the open source statistical software "R" (https://www.r-project.org) using the XCMS package. Univariate and multivariate feature selection algorithms were employed to determine subsets of potentially discriminative metabolic features. The moderated t-test was used for assessing univariate associations, while multivariable analyses included Orthogonal Projections to Latent Structures Discriminant Analysis (OPLS-DA) and signature identification through the Just Add Data (JAD) dataanalysis pipeline (Gnosis Data Analysis, Heraklion, Greece http://www.gnosisda.gr/). The JAD pipeline highlighted (i) one metabolic feature that could discriminate cancer-free controls from future squamous cell carcinoma patients (Area under the ROC Curve $[$ AUC-ROC] $=0.843$ ) and (ii) a signature consisted of 3 metabolic features, discriminating controls from adenocarcinoma lung cancer patients (AUCROC $=0.713)$. This relatively small pilot study identified putative biomarker signatures from circulating serum metabolites, specific for risk of lung adenocarcinoma and squamous cell carcinoma, which, could be potentially employed for the development of non-invasive detection assays for early lung cancer diagnosis.

Conflict of interest: CC has no conflict of interest. ODR has received honorary for one talk from Novartis, and funded in part by the Liaison Committee between the Central Norway Regional Health Authority (RHA) and the NTNU.

\section{References}

1. Krokstad S, Langhammer A, Hveem K, Holmen TL, Midthjell K, Stene TR, et al. Cohort Profile: the HUNT Study, Norway. Int J Epidemiol. 2013;42:968-77.

\section{D2-06.}

\section{Mesothelioma early detection and prevention-are we there yet?}

\section{Haining Yang ${ }^{1}$}

${ }^{1}$ University of Hawaii, Honolulu, HI, USA

Development of malignant mesothelioma has been linked to exposure to asbestos, erionite, and other carcinogenic mineral fibers (1). The mechanisms of asbestos-induced carcinogenesis are unclear, and the paradoxical observation that asbestos, a mineral fiber that causes cell death, can lead to malignant transformation is largely unexplained. Haining
Yang updated the group on the status of diagnostic and prognostic biomarkers for malignant mesothelioma (2) and reviewed some of the key mechanisms of asbestos-induced carcinogenesis that may begin to unravel mesothelioma pathogenesis (3). Cell necrosis induced by asbestos causes the release of a critical factor called High Mobility Group Box 1 protein (HMGB1). This protein functions as the "master switch" that kick-starts a series of inflammatory responses. Over time this leads to malignant transformation of mesothelial cells and mesothelioma development (4). Mesothelioma cells actively secrete HMGB1 and require it for tumor growth and progression. Therefore, targeting HMGB1 could be a novel therapeutic strategy for mesothelioma (5, 6). Moreover, mesothelioma patients and asbestos-exposed individuals have significantly higher HMGB1 levels compared to heavy smokers or healthy people. Thus, HMGB1 and its specific isoforms are sensitive and specific biomarkers to detect asbestos exposure and to identify mesothelioma patients $(5,7)$.

Conflict of interest: HY has grants from NIH, DoD and patents related to HMGB1.

\section{References}

1. Yang H, Testa JR, Carbone M. Mesothelioma epidemiology, carcinogenesis, and pathogenesis. Curr Treat Options Oncol. 2008;9:147-57.

2. Chen Z, Gaudino G, Pass HI, Carbone M, Yang H. Diagnostic and prognostic biomarkers for malignant mesothelioma: an update. Transl Lung Cancer Res. 2017;6:259-69.

3. Carbone M, Yang H. (2012). Molecular pathways: targeting mechanisms of asbestos and erionite carcinogenesis in mesothelioma. Clin Cancer Res. 18: 598-604. PMCID: PMC3291331.

4. Yang H, Rivera Z, Jube S, Nasu M, Bertino P, Goparaju C, et al. Programmed necrosis induced by asbestos in human mesothelial cells causes highmobility group box 1 protein release and resultant inflammation. Proc Natl Acad Sci USA. 2010;107: 12611-16.

5. Jube S, Rivera Z, Bianchi ME, Powers A, Wang E, Pagano IS, et al. Cancer Cell secretion of the DAMP protein HMGB1 supports progression in malignant mesothelioma. Cancer Res. 2012;72:3290-3301. PMCID: PMC3389268. (This paper made the cover of the July $1^{\text {st }} 2012$ issue of Cancer Research)

6. Yang H, Pelegrini L, Napolitano A, Giorgi C, Jube S, Preti A, et al. Aspirin delays mesothelioma growth by inhibiting HMGB1-mediated tumor progression. Cell Death Dis. 2015 Jun 11;6:e1789. PMID: 26068794.

7. Napolitano A, Antoine DJ, Pellegrini L, Baumann F, Pagano I, Pastorino S, et al. HMGB1 and Its hyperacetylated isoform are sensitive and specific serum biomarkers to detect asbestos exposure and to 
identify mesothelioma patients. Clin Cancer Res. 2016;22:3087-96.

D2-07.

\section{Extracellular small RNAs in human plasma and serum: clinical utility}

\author{
Klaas Max ${ }^{1}$ \\ ${ }^{I}$ The Rockefeller University, New York, NY, USA
}

Circulating extracellular ribonucleic acids (exRNA) are being pursued as potential liquid biopsy biomarkers for many diseases (1). However, lack of standardized exRNA isolation methods optimized for biofluids continues to hinder progress towards comprehensive profiling and clinical implementation. Klaas Max, from Thomas Tuschl's Laboratory, described their efforts to develop protocols for automated isolation of exRNA from urine and plasma/ serum to facilitate automated parallel processing of hundreds of clinical samples, followed by characterization of cell-free blood exRNA composition as a reference for biomarker studies (2). Extracellular ribonucleic acid was isolated from several hundered cell-free biofluid samples (serum, plasma, and urine) of patient cohorts including renal failure, cardiovascular disease, allograft recipients, cancer patients, and normal individuals. Using optimized protocols, they recovered nanogram quantities of exRNA per sample at high purity for direct use in complementary deoxyribonucleic acid (cDNA) library preparation. The exRNA biofluid composition was characterized using barcoded small RNA cDNA library preparation in batches of 24 RNA samples, followed by single-end Illumina HiSeq sequencing yielding up to 150 million reads per batch and lane. Sequence reads were demultiplexed and mapped against a curated human reference transcriptome to obtain miRNA read frequency profiles as well as an abundance of fragments of other RNA classes, such as transfer RNAs (tRNAs), small nuclear RNAs (snRNAs), small conditional RNAs (scRNAs), ribosomal RNAs (rRNAs), messenger RNAs (mRNAs), and long non-coding RNAs (lncRNAs). Sequencing data was used for differential analysis of miRNA abundance, which allowed identification of indicators discriminating disease subcategories and diseaseassociated conditions. Small RNA (sRNA)-derived cDNA library sequencing helped to define normal sRNA variability and revealed organ-specific signatures in certain patient cohorts. Furthermore, a unique exRNA phenotype stable for over a year was observed in one of the volunteers characterized by two-fold total miRNA levels and $>20$-fold elevated endocrine-cell-specific miRNAs in serum and plasma. "External standardization helped to detect quantitative differences in erythrocyte and platelet-specific miRNA contributions and miRNA concentrations between biofluids" (2).

Conflict of interest: KM holds a patent "Method of RNA isolation from clinical samples" US 15/540,528.

\section{References}

1. Li K, Rodosthenous RS, Kashanchi F, Gingeras T, Gould SJ, Kuo LS, et al. Advances, challenges, and opportunities in extracellular RNA biology: insights from the NIH exRNA Strategic Workshop. JCI Insight. 2018;3.

2. Max KEA, Bertram K, Akat KM, Bogardus KA, Li J, Morozov $\mathrm{P}$, et al. Human plasma and serum extracellular small RNA reference profiles and their clinical utility. Proc Natl Acad Sci USA. 2018;115: E5334-E5343.

\section{D2-08.}

\section{Precision medicine: prognostic and predictive biomarkers}

\section{Implementation of precision oncology: strategies to overcome challenges}

\section{Apostolia-Maria Tsimberidou ${ }^{1}$}

${ }^{I}$ The University of Texas MD Anderson Cancer Center, Houston, TX, USA

Precision Medicine currently refers to the use of therapeutic agents that target any biological abnormality associated with carcinogenesis, including genomic, transcriptional, proteomic, epigenetic aberrations and immune mechanisms. Apostolia-Maria Tsimberidou provided an overview of the early days of molecular-testing guided therapy. In 2007, the first personalized medicine program for patients with advanced cancer was initiated, the IMPACT (Initiative for Molecular Profiling and Advanced Cancer Therapy). Matched/targeted therapy compared with non-matched therapy showed improved progression-free survival, overall survival and response rates (1). Landmark analyses demonstrated that patients with one molecular alteration who received targeted therapy and had an objective response had longer progression-free survival compared to those who did not achieve response. An ongoing randomized study, IMPACT2, evaluates molecular profiling and targeted agents in patients with metastatic cancer (2). Challenges in precision medicine include the large amount of complex data from tumor/cell-free DNA profiling, exome sequencing, proteomics, RNA analysis and immune markers that require accurate bio-informatic analysis and interpretation of the results, careful treatment 
selection and treatment optimization during monitoring of evolving molecular abnormalities. Cancer immunotherapy in implementation of precision oncology is thought to overcome the complexity of molecular profiling, by initiating an immune response against patients' tumors. The approval of pembrolizumab for solid tumors with microsatellite instability (MSI)-high, and the success of Tcell therapy prompted the development of multiple clinical trials of immunotherapy. Innovative biomarker-driven clinical trials, " $\mathrm{N}$ of 1 " databases and the use of artificial intelligence hold the promise to expedite precision oncology (3).

Conflict of interest: Apostolia Tsimberidou has served in the boards of Genentec, ASCO CRC, ASCO ImmunoOncology, at the Exactis Expert Panel Meeting, the WIN Consortium Collaboration, honoraria as invited speaker for Roche Europe, Covance and Genetech and grant support by CPRIT, EMD Serono, Inc., Boston Biomedical, Verastem Inc./Placon Ther, Inc., Karus Therapeutics, Tvardi Therapeutics, Immatics Biotechnologies, OBI Pharma, Parker Institute for Cancer Immunotherapy, Baxter Healthcare Corp., Onyx Pharmaceuticals, Foundation Medicine, Inc, Bayer Healthcare and Tempus. A-MT also receives royalties for a book on targeted therapy and cancer research.

\section{References}

1. Tsimberidou AM, Hong DS, Ye Y, Cartwright C, Wheler JJ, Falchook GS, et al. Initiative for molecular profiling and advanced cancer therapy (IMPACT): an md anderson precision medicine study. JCO Precis Oncol. 2017;2017.

2. Tsimberidou AM. Initiative for molecular profiling and advanced cancer therapy and challenges in the implementation of precision medicine. Curr Probl Cancer. 2017;41:176-81.

3. Fountzilas E, Tsimberidou AM. Overview of precision oncology trials: challenges and opportunities. Expert Rev Clin Pharmacol. 2018;11:797-804.

\section{D2-09.}

\section{Circulating tumor cells and circulating micro ribonucleic acids (miRNA) in breast cancer}

\section{Sofia Agelaki \\ ${ }^{1}$ University of Crete, Heraklion, Greece}

Metastasis is the leading cause of breast cancer (BC) related death. Stratification of patients according to prognosis could lead to a personalized treatment approach and improved survival. To this end, identification of markers predictive of metastasis and patient outcome are in need. Blood-based biomarkers are easily accessible and attract significant interest in the field of biomarker research. Sofia Agelaki described clinical research focused on development and validation of blood-based biomarkers of $\mathrm{BC}$ metastatic dissemination. Circulating tumor cells (CTC) are the surrogates of micrometastatic disease and precursors of distant metastasis. Their prognostic significance has been demonstrated both in adjuvant and metastatic BC (1). Beyond detection and enumeration, the characterization of single CTCs can contribute to a better understanding of the metastatic process, improve patient prognostication and treatment monitoring. Characterization of CTCs allows the identification of subpopulations with differential prognostic relevance in BC (2). Specifically, insulin-like growth factor1 receptor (IGF1R) expression is commonly observed in CTCs of BC. The IGF1R(+) CTC number decreases significantly in metastatic compared to early disease. IGF1R and epithelial calcium-dependent adhesion (E-cadherin) are co-expressed at the single CTC level, and the transition from early to metastatic $\mathrm{BC}$ is associated with a reduction in the expression of both molecules on CTCs. Accordingly, $\operatorname{IGF} 1 \mathrm{R}(+)$ CTCs were associated with a favorable outcome in early BC (3). In metastatic disease the detection of CTCs co-expressing putative cancer stem cell (CSC) and epithelial-to-mesenchymal transition (EMT) markers is associated with aggressive disease characteristics and worse outcome in patients undergoing first-line chemotherapy ${ }^{4}$. Moreover, chemotherapy enriched the CSC/EMT phenotype of CTCs and this was associated with human epidermal growth factor receptor 2 (HER2)-negative status and disease progression. Circulating miRNAs have also attracted significant interest as liquid-based biomarkers. The expression of plasma microRNAs (miR-23b, miR-190, miR-21, miR-200b and miR-200c) involved in tumor dormancy, EMT and metastatic dissemination discriminate between early and metastatic disease stage in BC (5). Furthermore, their expression holds prognostic significance in patients with metastatic disease treated with first-line chemotherapy. Differential expression of these miRNAs is evident before the initiation of adjuvant therapy between patients with early BC who will experience subsequent disease relapse compared to non-relapsed patients. Moreover, differential expression of miRNAs is also observed among patients with early versus late onset of metastasis years before its clinical detection. The above results support the clinical relevance of CTC characterization and metastasis promoting miRNAs for the prediction of tumor dissemination and the refinement of prognosis of patients with $\mathrm{BC}$.

Conflict of interest: This work was partly supported by the Hellenic Society of Medical Oncology (HESMO) and the Anticancer Research Support Association (ARSA).

\section{References}

1. Bidard FC, Peeters DJ, Fehm T, Nole F, GisbertCriado R, Mavroudis D, et al. Clinical validity of 
circulating tumor cells in patients with metastatic breast cancer: a pooled analysis of individual patient data. Lancet Oncol. 2014;15 406-14.

2. Agelaki S, Dragolia M, Markonanolaki H, Alkahtani S, Stournaras C, Georgoulias V, et al. Phenotypic characterization of circulating tumor cells in triple negative breast cancer patients. Oncotarget. 2017;8:5309-22.

3. Spiliotaki M, Mavroudis D, Kokotsaki M, Vetsika EK, Stoupis I, Matikas A, et al. Expression of insulinlike growth factor-1 receptor in circulating tumor cells of patients with breast cancer is associated with patient outcomes. Mol Oncol. 2018;12:21-32.

4. Papadaki MA, Stoupis G, Theodoropoulos PA, Mavroudis D, Georgoulias V, Agelaki S. Circulating tumor cells with stemness and epithelial-tomesenchymal transition features are chemoresistant and predictive of poor outcome in metastatic breast cancer. Mol Cancer Ther. 2019;18:437-47.

5. Papadaki C, Stratigos M, Markakis G, Spiliotaki M, Mastrostamatis G, Nikolaou C, et al. Circulating microRNAs in the early prediction of disease recurrence in primary breast cancer. Breast Cancer Res. 2018;20:72.

\section{D2-10.}

\section{Cancer diagnosis by machine learning-powered RNA- sequencing of tumor-educated platelets}

\section{Jordi Berenguer ${ }^{1}$ \\ ${ }^{1}$ Pangaea Oncology. Quiron Dexeus University Hospital, Barcelona, Spain}

During the last century, the field of cancer diagnostics has experienced several revolutions. In a few decades, cancer diagnosis has evolved from histopathological classification of tumor biopsies to non-invasive, ultrasensitive molecular techniques. Continuing technological improvements are starting to enable the diagnosis of tumors by capturing tumor-derived material present in different liquid biosources, such as blood, urine or saliva, in what is commonly referred to as liquid biopsies. "Classical" liquid biopsies aim to detect the presence of biomarkers, either over- or underexpressed sets of genes, or genetic aberrations such as point mutations or gene fusions, which inform treatment decisions. Given the ease of obtaining liquid biopsies, these biomarkers can be monitored longitudinally over time on the same patient, so the treatment can be more precisely tuned in response to the changing levels of these molecules. Different biosources have been successfully obtained from liquid biopsies, including cell-free DNA and RNA, circulating tumor cells, extracellular vesicles (including exosomes, shed microvesicles and apoptotic bodies) and, more recently, blood platelets (1). Jordi Berenguer discussed exciting new applications of using blood platelets for early detection of cancer. Platelets are known to act as vacuum cleaners in the blood, ingesting tumor-derived material, which can be detected and analysed. For instance, of echinoderm microtubule-associated protein-like 4 and anaplastic lymphoma kinase (EML4-ALK) fusions can be detected in platelets of lung cancer patients with high precision, and this information is highly valuable to decide whether the patient should be treated with crizotinib (2). Recent publications on platelet-based cancer diagnostics have resulted in a paradigm change: platelets, which don't have a nucleus, interact with tumors and, in turn, change their splicing patterns, becoming what we call tumor-educated platelets (TEPs). Deep sequencing of platelets and TEPs revealed that their landscape of spliced transcripts reflects the characteristics of the tumor. By the combination of deep sequencing and machine learning-based algorithms to cluster platelet samples in an unsupervised fashion, platelets facilitate pancancer diagnostics, tumor type and grade classification and molecular pathway identification with high accuracy (3). It is important to note that the algorithms are able to classify tumors according to their genetic aberrations indirectly, that is, not finding the aberration per se, but the reaction of the TEPs.

Conflict of interest: This project has received funding from the European Union's Horizon 2020 research and innovation programme under the Marie Skłodowska-Curie grant agreement No 712949 (TECNIOspring PLUS) and from the Agency for Business Competitiveness of the Government of Catalonia.

\section{References}

1. Bracht JWP, Mayo-de-Las-Casas C, Berenguer J, Karachaliou N, Rosell R. The Present and Future of Liquid Biopsies in Non-Small Cell Lung Cancer: Combining Four Biosources for Diagnosis, Prognosis, Prediction, and Disease Monitoring. Curr Oncol Rep. 2018;20:70.

2. Nilsson RJ, Karachaliou N, Berenguer J, GimenezCapitan A, Schellen P, Teixido C, et al. Rearranged EML4-ALK fusion transcripts sequester in circulating blood platelets and enable blood-based crizotinib response monitoring in non-small-cell lung cancer. Oncotarget. 2016;7:1066-75.

3. Best MG, Sol N, Kooi I, Tannous J, Westerman BA, Rustenburg F, et al. RNA-seq of tumor-educated platelets enables blood-based pan-cancer, multiclass, and molecular pathway cancer diagnostics. Cancer Cell. 2015;28:666-76. 
D2-11.

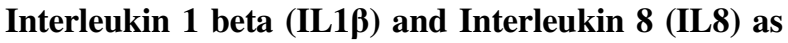 biomarkers and mediators of chemo-resistance in malignant pleural mesothelioma}

Vladan Milosevic ${ }^{1}$, Chiara Riganti ${ }^{1}$

${ }^{1}$ University of Turin, Turin, Italy

Standard therapy in malignant pleural mesothelioma (MPM) induces only partial response in less than half of the patient and no response in the rest due to the intrinsic chemoresistance. Tumor-derived stem cells are responsible for MPM dissemination and progression, but it is not known wether they play a role in chemoresistance of this tumor. At the moment, there are no specific biomarkers predictive of chemo-response in MPM. Vladan Milosevic discussed a study focused on investigating the mechanisms by which MPM stem cells may engender chemoresistant properties as well as identifying potential biomarkers of chemoresistance. MPM cell lines were generated from biopsies and pleural effusions of MPM patients. The stem cell component was isolated by sorting the SRY-box transcription factor 2, octamer 4, nanog homebox and high aldehyde dehydrogenase ( $\mathrm{SOX}_{2}{ }^{+} \mathrm{Oct}_{4}{ }^{+} \mathrm{Nanog}^{+} \mathrm{ALDH}^{\text {bright }}$ ) cells and then confirming their clonogenicity and self-renewal potential. MPM stem cells showed higher resistance to cisplatin and pemetrexed compared to non stem cells. High-throughput polymerase chain reaction (PCR) array was used to examine expression of interleukin genes. Higher expression of a vast number of cytokines, particularly of interleukin 1 beta (IL1 $\beta$ ) and interleukin 8 (IL8), was observed in MPM stem cells. Targeted Knock-out of ILI $\beta$ and IL8 in MPM stem cells using Clustered Regularly Interspaced Short Palindromic Repeats (CRISPR) and CRISPR associated system (CAS) restored chemosensitivity to cisplatin and pemetrexed. IL1 $\beta$ and IL8 up-regulated ABCB5, a drug efflux transporter, in MPM stem cells. Consistently, elevated levels of these two cytokines were observed in cells derived from patients non-responsive to therapy. This study showed that MPM stem cells are resistant to a broad spectrum of effects of first-line drugs (such as cisplatin and pemetrexed). Knock-out experiments suggested that IL1- $\beta$ and IL-8 autocrine stimulation induced resistance to cytotoxic effects in MPM stem cells, by upregulating ABCB5. Furthermore, detection of both IL1- $\beta$ and IL-8 production in patientderived cells could predict therapy response.

Conflict of interest: VM and CR declare that they have no conflict of interest. CR received grant support from Italian Assciation for Cnacer Research AIRC, and Cassa di Rispoarmio di Torino.
D2-12.

Lung cancer biomarkers: Beyond Genomics

Ana I. Robles ${ }^{1}$

National Cancer Institute, Bethesda, MD, USA

Widespread implementation of lung cancer screening is creating a need for better clinical management options for patients diagnosed with early stage disease. Up to $30 \%$ of surgically treated Stage I lung cancer patients will suffer recurrence within 5 years of diagnosis. Ana Robles described ongoing collaborative efforts to generate comprehensive 'omics' profiles for a large cohort of biospecimens from Stage I lung cancer patients with well-curated epidemiological and clinical data at the Laboratory of Human Carcinogenesis, NCI (Figure 1). Included are the profiling of genomic, epigenomic, transcriptomic, and metabolomic landscapes, and, more recently, the microbiome of single patients. These rich datasets are being mined to identify diagnostic and prognostic biomarkers that show evidence for statistically significant risk separation and improved predictive value (1). Specifically, Homeobox protein A9 (HOXA9) promoter methylation has been identified and validated as a prognostic biomarker for patients with Stage I lung adenocarcinoma (2). Robles and colleagues have now developed and optimized an assay that utilizes Droplet Digital PCR (ddPCR) technology to assess HOXA9 promoter methylation in Formalin-Fixed Paraffin-Embedded (FFPE) tissues routinely generated in the clinic (3). The group, led by Curt Harris, also recently conducted the first comprehensive characterization of the lung cancer microbiome. Evidence from 16S ribosomal RNA (rRNA) and metagenomics analysis indicated the presence of specific bacterial strains in lung tumors (4). Ongoing animal studies will evaluate how these bacteria affect the lung immune microenvironment and the rate of tumor development. In addition, Machine Learning strategies are being applied to the integrative analysis of these multi-dimensional datasets. The integrative analysis of 'omics' data may provide insight on the early steps of carcinogenesis and a framework to identify clinically relevant biomarkers and probe their mechanistic association with disease.

Conflict of interest: AR has no conflicts of interest.

\section{References}

1. Robles AI, Harris CC. Integration of multiple "OMIC" biomarkers: a precision medicine strategy for lung cancer. Lung Cancer. 2017;107:50-8.

2. Robles AI, Arai E, Mathe EA, Okayama H, Schetter AJ, Brown D, et al. An integrated prognostic classifier for stage I lung adenocarcinoma based on mRNA, microRNA, and DNA methylation biomarkers. J Thorac Oncol. 2015;10:1037-48. 
3. Lissa D, Ishigame T, Noro R, Tucker MJ, Bliskovsky $\mathrm{V}$, Shema $\mathrm{S}$, et al. HOXA9 methylation and blood vessel invasion in FFPE tissues for prognostic stratification of stage I lung adenocarcinoma patients. Lung Cancer. 2018;122:151-59.

4. Greathouse KL, White JR, Vargas AJ, Bliskovsky $\mathrm{VV}$, Beck JA, von Muhlinen $\mathrm{N}$, et al. Interaction between the microbiome and TP53 in human lung cancer. Genome Biol. 2018;19:123.

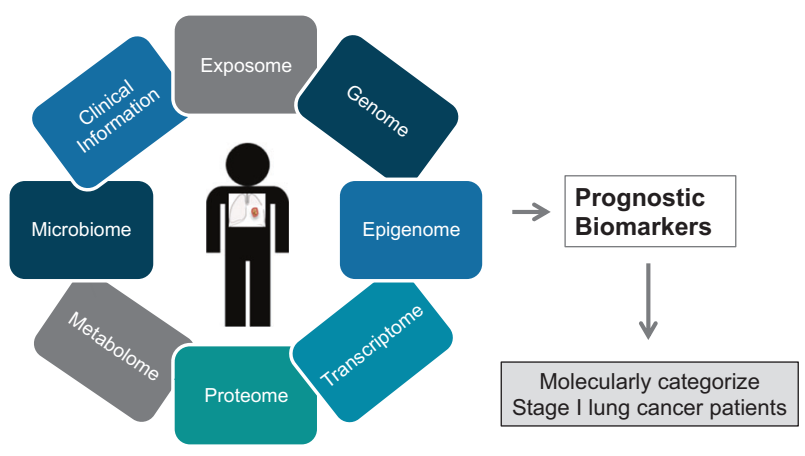

Figure 1 Clinically useful biomarkers of early stage lung cancer can be identified from the integrative analysis of large-dimensional data

\section{D2-13.}

\section{How to identify aggressive from indolent prostate cancer?}

\section{May-Britt Tessem ${ }^{1}$ \\ ${ }^{I}$ Norwegian University of Science and Technology, Trond- heim, Norway}

Overtreatment in prostate cancer (Pca) is a burden for health care economy and for quality of life. Correct diagnosis of early stage Pca is challenging given the limitations of the currently available clinical tools and the biological understanding of Pca. There is an urgent need for a reliable diagnostic tool to identify men whose Pca is likely to be most aggressive and life threatening. Cutting-edge 'omics' technologies (metabolomics, transcriptomics, proteomics and genomics) are moving at a breath-taking pace. These techniques have the potential to stratify disease and individualize cancer care, and are thus, a promising choice to advance diagnostics. Moreover, they become especially powerful if applied directly to human tissue samples in order to capture the complexity that is lost in cell lines and animal models. May-Britt Tessem described an approach to Pca biomarker identification based on metabolomics analyses (MR spectroscopy) on prostate tissues coupled with histopathology, gene expression and immunohistochemistry (1). Using this approach, they examined concentrations of several potential biomarkers for Pca, including citrate and polyamines (2). The concentrations of these were significantly decreased in malignant aggressive disease, in patients positive for the transmembrane protease, serine 2 (TMPRSS)ERG gene fusion, and in those carrying a novel Wnt5a signature connected to endothelial mesenchymal transition (EMT), the latter of which is associated with recurrent Pca. Additionally, concentrations of citrate and spermine predicted Pca recurrence. Thus, these biomarkers are highly promising as a tool for clinical treatment decisions. The clear molecular mechanism accounting for low levels of polyamines and citrate in human Pca is being debated and these markers are yet to be validated in the clinic. In part, this may be due to the challenge of tissue heterogeneity and the mix of cell types found in tissue analyses. In the future, a protocol that uses multi-omics and spatial information from a single tissue sample may be needed to explore the clinical relevance of the suggested molecular mechanisms in large patient cohorts (3). The understanding of such biomarkers can personalize Pca diagnosis and open up new treatment strategies.

Conflict of interest: MBT has no conflict of interest.

\section{References}

1. Euceda LR, Andersen MK, Tessem MB, Moestue SA, Grinde MT, Bathen TF. NMR-based prostate cancer metabolomics. Methods Mol Biol. 2018;1786: 237-57.

2. Braadland PR, Giskeodegard G, Sandsmark E, Bertilsson H, Euceda LR, Hansen AF, et al. Ex vivo metabolic fingerprinting identifies biomarkers predictive of prostate cancer recurrence following radical prostatectomy. Br J Cancer. 2017;117:1656-64.

3. Andersen MK, Rise K, Giskeodegard GF, Richardsen $\mathrm{E}$, Bertilsson $\mathrm{H}$, Storkersen $\mathrm{O}$, et al. Integrative metabolic and transcriptomic profiling of prostate cancer tissue containing reactive stroma. Sci Rep. 2018;8:14269.

\section{D2-14.}

\section{Blood vessel function as a biomarker for susceptibility to nanomedicines}

\section{Einar Sulheim ${ }^{I}$}

${ }^{I}$ Norwegian University of Science and Technology, Trondheim, Norway

Preclinical research has shown that nanoparticles and macromolecules can accumulate in solid tumors due to the enhanced permeability and retention effect, and give 
therapeutic efficacy superior to the standard formulation of the drug. However, not all tumor models respond equally well to nanomedicines, and clinical trials with nanomedicines typically fail to show improved efficacy across an unstratified patient group. Tools that can be used to preselect patients for nanomedicines would be valuable. Einar Sulheim investigated whether imaging of the tumor vasculature in xenograft models could be used to predict nanoparticle accumulation and hence serve as a biomarker for the use of nanomedicines (1). Five different tumor models were grown subcutaneously in athymic mice and imaged with X-ray micro-computed tomography $(\mu \mathrm{CT})$, diffusion weighted and dynamic contrast enhanced magnetic resonance imaging (MRI), and contrast-enhanced ultrasound (Figure 1). Parameters obtained from the in vivo imaging were then evaluated for correlation with the accumulation of $100 \mathrm{~nm}$ polystyrene nanoparticles. The tumors were also characterized for density of blood vessels and collagen by microscopy of tumor sections. It was found that nanoparticle accumulation correlated with vascular properties. The blood vessel quality, evaluated through the inflow time in the time-intensity curves from contrastenhanced ultrasound imaging, correlated significantly $(p=$ 0.041) with nanoparticle accumulation. This indicates that chaotic vasculature with shunts and dead ends that give microbubble retention also is more susceptible to nanoparticle accumulation. The vascular density, when quantified by microscopy of lectin-perfused blood vessels also correlated significantly $(p=0.0056)$ with the accumulation of nanoparticles, but this was not captured by the in vivo imaging, probably due to lack of sensitivity towards the smaller vessels. This study indicates that imaging or measurements of the vasculature in tumors could be a useful alternative to theranostics or companion nanoparticles for stratifying patients for therapy with nanomedicines and that blood vessel architecture, and not only leakiness, could be an important factor for nanoparticle accumulation.

Conflict of interest: ES has received grant support from the Norwegian Cancer Society.

\section{Reference}

1. Sulheim E, Kim J, van Wamel A, Kim E, Snipstad S, Vidic I, et al. Multi-modal characterization of vasculature and nanoparticle accumulation in five tumor xenograft models. J Control Release. 2018; 279:292-305.

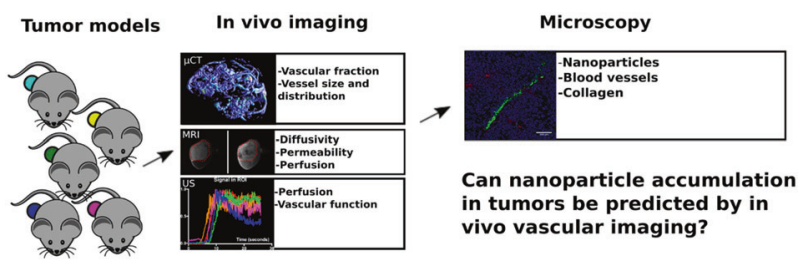

Figure Characterization of five xenograft tumor models to evaluate nanoparticle accumulation

\section{Resources in the service of precision cancer treatment}

D2-15.

Open access to human gene, proteomics and metabolomics data (TCGA); Escalating biomarker research or not?

\section{Jean Claude Zenklusen ${ }^{1}$}

\section{${ }^{I}$ National Cancer Institute, Bethesda, MD, USA}

The Cancer Genome Atlas (TCGA) is a joint project of the National Cancer Institute (NCI) and the National Human Genome Research Institute (NHGRI). Jean Claude Zenklusen, TCGA Director, gave an overview of the project, the largest-scale cancer genomics project to date, and its lasting impact. Begun in 2006 as a pilot project, TCGA collected, in the following 7 years, more than 11,000 cases across 33 tumor types, and generated a vast, comprehensive data set using a variety of technologies to investigate DNA, RNA and protein to investigate the molecular changes that occur in cancer. Tissue sample collection and data generation were completed in 2013 and 2016, respectively. Integrative cross-platform analyses of TCGA data on individual cancer types have been published for all of the tumor types (https://cancergenome.nih.gov/publications) (1). The value of the TCGA dataset cannot be overstated (2). In the last decade, the data and publications have become the standard reference for studies in the tumors characterized by the program. Its richness has enabled researchers to use it as the preliminary dataset to generate new hypothesis to further investigate the role of the genomic alterations found. TCGA has also allowed to define a more meaningful taxonomy of cancer types and subtypes, based not on location but biological characteristics, thus enabling more rational treatment course. Surprisingly, the TCGA data has been used in ways that were not envisioned at the onset of the program, such as the mining of the data to discover new viruses and other microbial agents. However, translation to the clinic of the wealth of information gathered during the project has been modest, mainly for the lack of extensive clinical data and outcomes, but also because we have not been able to truly comprehend the complexity of the biological processes that underlie cancer, focusing mainly on single gene mutations that are a poor reflection of the biological reality. 


\section{References}

1. Blum A, Wang P, Zenklusen JC. SnapShot: TCGAAnalyzed Tumors. Cell. 2018;173:530.

2. Hutter C, Zenklusen JC. The Cancer Genome Atlas: Creating Lasting Value beyond Its Data. Cell. 2018;173:283-85.

Conflict of interest: JCZ has no conflict of interest. D2-16.

\section{HUNT Biobank-a unique resource for biomarker discovery and validation}

\section{Kristian Hveem ${ }^{1}$ \\ ${ }^{1}$ Norwegian University of Science and Technology, Trond- heim, Norway}

The HUNT biobank is the biorepository of the NordTrøndelag Health Study (HUNT), a prospective, longitudinal population-based cohort study in central Norway. Kristian Hveem, HUNT Biobank Director, gave an overview of this biobank developed over four decades. Based on the HUNT study, a variety of biological samples have been collected from a total of 100,000 study participants, 70,000 presently chip-genotyped and imputed up to 30 million markers. Individual health data, data on exposure and from clinical examinations are available since 1984 in the form of four phases of the HUNT study, 10 years apart, all of them validated, quality-controlled and stored in the HUNT databank and HUNT data center. In 2006, the HUNT biobank was established as a modern state-of-the-art facility, with fully automated procedures for sample handling, storage and retrieval, holding several millions of sample aliquots. In 2013, the HUNT biobank was recognized as the European Research Biobank of the year by the European Society of Biopreservation and Biobanking (https://esbb.org/). Using a personal identification number, incident clinical phenotypes have been collected from a number of local, regional and national registries as well as electronic health records. This prospective design, with multiple time-points, creates a unique research platform for both biomarker discovery and validation based on metabolites, protein arrays, singlenucleotide polymorphisms (SNP), micro RNA, and others.

Conflict of interest: $\mathrm{KH}$ has received grant support from K. G. Jebsen Stiftelsen.

\section{D2-17.}

\section{Why is precision medicine so difficult? Conclusions and thoughts}

Ana I. Robles ${ }^{1}$

${ }^{I}$ National Cancer Institute, Bethesda, MD, USA

The fundamental premise of Precision Oncology is the ability to match therapy to an individual's tumor. This "tailored" approach is based on the belief that knowledge of the molecular characteristics of tumors will enable oncologists to identify specific, and sometimes unique, weaknesses that can be targeted therapeutically. The proven success of this strategy in identifying subsets of patients with clinically actionable abnormalities, coupled with the development of increasingly affordable sequencing technologies, has resulted in genomic testing rapidly becoming if not routine, at least an expected part of cancer patient clinical management. The poster child for this approach is NSCLC, where testing for predictive mutations is now standard of care. On the other hand, predictive biomarkers of response to immunotherapy are proving to be complex and context-dependent, and it appears that multiple cellular components as well as intrinsic molecular features of tumors will have to be interrogated. Consortia-driven projects led by The Cancer Genome Atlas (1) have generated comprehensive catalogues of genomic alterations in multiple tumor types and identified many that can potentially be targeted therapeutically. Similarly, the community-based Norwegian HUNT Biobank (2) provides biospecimens and rich clinical data with decades of followup, a unique source for discovery of early diagnostic markers. These datasets provide a rich source for biomarker identification and validation in support of translational efforts.

Conflict of interest: AR has no conflict of interest.

\section{References}

1. Blum A, Wang P, Zenklusen JC. SnapShot: TCGAAnalyzed Tumors. Cell. 2018;173:530.

2. Krokstad S, Langhammer A, Hveem K, Holmen TL, Midthjell K, Stene TR, Bratberg.

G, Heggland J, Holmen J. Cohort Profile: the HUNT Study, Norway.

Int J Epidemiol. 2013 Aug;42(4):968-77. https:// doi.org/10.1093/ije/dys095. Epub 2012 Aug 9. 